\title{
Thermal properties of the three-dimensional graphene/paraffin nanocomposite phase change materials
}

\author{
Jing $\mathrm{Li}^{1,2^{*}}$, Yanning Liao ${ }^{1,2}$, Shaowei $\mathrm{Li}^{1,2}$, Xu Yang $^{1,2}$, and Naixun $\mathrm{Jiao}^{1,2}$ \\ ${ }^{1}$ Key Laboratory of Low-grade Energy Utilization Technologies and Systems (Chongqing University), \\ Ministry of Education, Chongqing, 400044, China \\ ${ }^{2}$ School of Energy and Power Engineering, Chongqing University. Chongqing, 400044, China
}

\begin{abstract}
The excellent properties of graphene phase change nanocomposite made it have potential application value in the field of heat storage materials, which was expected to achieve the integration of heat transfer and storage. In order to enhance the thermal performance of paraffin in energy storage, the structure models of n-octadecane and three kinds of graphene/n-octadecane composites were established. Molecular dynamics method was used to study the variation of thermophysical properties. It is found that the strong interaction between graphene and noctadecane restricts the diffusion intensity of n-octadecane molecules, which reflects in the decreasing trend of the self-diffusion coefficient. In addition, the thermal conductivity of each system in the solid state is higher than that of liquid, and abruptly drops near the melting point. The thermal conductivity of the composite PCM always higher than the pure noctadecane and increases with the amount of graphene.
\end{abstract}

\section{Introduction}

Nowadays, limited reserves of fossil fuels and excessive emissions of greenhouse gases make efficient use of energy a key problem to be solved. Among various forms of energy, thermal energy is widely spread in nature as in solar radiation, geothermal energy, industrial waste heat and building applications. Using phase change materials (PCMs) for thermal energy storage, can not only reduce the mismatch between supply and demand, but also improve the performance and reliability of energy distribution networks [1-2]. Phase change materials (PCMs) can solve the problem of discontinuity and instability in solar energy supply in time and realize the efficient and rational use of energy, so as to solve the energy and environment crisis [3-5].

Among numerous PCMs, paraffin (saturated hydrocarbons with $\mathrm{C}_{\mathrm{n}} \mathrm{H}_{2 \mathrm{n}+2}$ formula), has proved to be desirable for thermal energy storage due to its rich latent heat, low cost, thermal and chemical stability, non-toxic and non-corrosive. However, paraffin suffers from inherently low thermal conductivity which affects the rate of thermal response [6-7]. Traditional techniques to enhance the thermal conductivity of PCM is to include metallic

*Corresponding author: 1j202740@cqu.edu.cn 
fins and utilization of metallic foams, while their incorporation in PCM is limited in many circumstances due to their chemical activity and high density [8-11]. The discovery of graphene, consisting of a single-layer of carbon atoms that are arranged in two-dimensional honeycomb structure, has gained considerable attention around the world owing to its extraordinary physical properties [12-17]. There is still a lack of deep insights into the microscopic behaviour of melting phenomena and the enhancing mechanism of thermal transport. Molecular dynamics (MD) simulation is powerful technique to solve the above problems.

In this work, we proposed a nanostructure of the three-dimensional graphene/paraffin nanocomposites, which consist of three-dimensional porous graphene networks and energy storage material paraffin. The thermal properties of the three-dimensional nanoporous graphene/paraffin nanocomposites during phase transition are investigated systematically via MD simulations. This work will be beneficial to the development of PCM at thermal energy storage in the future.

\section{Model and Method}

\subsection{Structure Model of Simulated systems}

We performed the MD simulations both for pure paraffin and three-dimensional nanoporous graphene/paraffin nanocomposites which are applied to represent paraffin molecule. In the simulation n-octadecane is applied to represent paraffin molecule. The three-dimensional nanoporous graphene/paraffin nanocomposites, as shown in Fig. 1 (a), the graphene nanosheets are dispersed into paraffin to form the three-dimensional nanoporous graphene/paraffin nanocomposites.

\subsection{Simulation method}

We used MD method to calculate the established models. COMPASS force field is chosen to describe the interactions among alkane and graphene system. Algorithm is used to optimize the geometry after each structure was constructed. Then 200 ps NPT ensemble and 100 ps NVT ensemble were used to equilibrate the system.

The thermal conductivity can be calculated using Fourier's law as:

$$
K=-\frac{J_{z}}{\left(\frac{\partial T}{\partial z}\right)_{\text {ave }}}
$$

Where $J z$ is the heat flux in the z-direction, and $\left(\frac{\partial T}{\partial z}\right)$ ave represents an average temperature gradient. The heat flux $J z$ can be calculated as:

$$
J_{z}=\frac{1}{2 A} \frac{\overline{\Delta E}}{\Delta T}
$$

Where $\Delta E$ is the change of the kinetic energy, $\Delta T$ is the time interval, $\mathrm{A}$ is the crosssectional area perpendicular to the flux direction.

\section{Results and discussion}

Generally, the maximum deviation of simulated density compared with theoretical value is lower than 5\%, which proves that the constructed amorphous structure is effective. 


\subsection{Mean square displacements and Self diffusion coefficients}

To rigorously characterize structural changes associated with the melting process and better demonstrate the phase change of n-octadecane and graphene/paraffin nanocomposites, we calculate mean square displacement (MSD) of atoms, which can be expressed as:

$$
\operatorname{MSD}(t)=\frac{1}{N}\left\langle\sum_{i=1}^{N}\left|r_{i}(t)-r_{i}(0)\right|^{2}\right\rangle
$$

where $r_{i}(t)$ is the position of the atom at the given time and $r_{i}(0)$ represents the initial position of the atom. According to the Einstein formula, the self diffusion coefficient can be calculated as:

$$
D=\frac{1}{6 N} \lim _{t \rightarrow \infty} \frac{d}{d t} \sum_{i \rightarrow j} N\left\langle\left|r_{i}(t)-r_{i}(0)\right|^{2}\right\rangle
$$

where $D$ is the self diffusion coefficient and $\mathrm{N}$ represents the number of atoms moving diffusively in the system.

Then, the self diffusion coefficient computed from the linear regime of the MSD curve of each model at different temperatures is plotted in Fig.1 (b), the self diffusion coefficient shows obvious change at about $300 \mathrm{~K}$ in the pure paraffin, which indicates that the phase transition of paraffin from solid to liquid is happened. For the composites, the self diffusion coefficients are all smaller than that of pure paraffin, and decrease as the mass fraction of graphene increases.

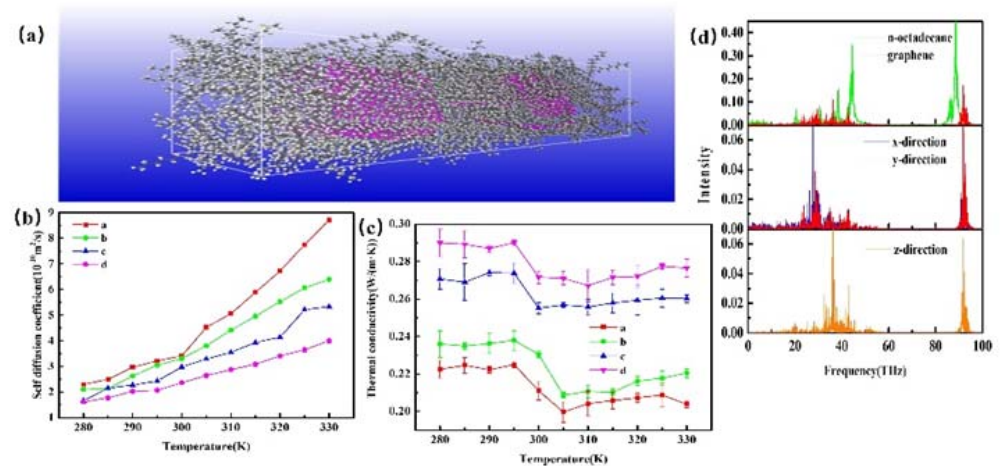

Fig. 1. (a) The structure of three-dimensional nanoporous graphene/paraffin nanocomposites. (b) Self- diffusion coefficients of pure paraffin and the composites at different temperatures. (c) Thermal conductivities of pure paraffin and the composites at different temperatures.(d) Vibrational power spectra of n-octadecane and graphene in composite system at equilibrium state of $280 \mathrm{~K}$.

\subsection{Thermal Conductivity and Velocity auto-correlation function}

The thermal conductivities of the four systems are presented in Fig. 1(c), which shows that the thermal conductivity varies with temperature and decreases sharply near the melting point. The reason for this phenomenon is that the phase change material is in a mixed state of solid and liquid near the phase transition point, the molecular movement is hindered in the solid-liquid boundary region and thermal energy is not easy to transfer between these regions. Constantly, the high thermal conductivity of graphene is more important to the enhancement of thermal conductivity of composites than the thermal resistance of solidliquid interface, which increasing of thermal conductivity of composites obviously. 
Thermal energy is essentially the energy of atomic vibration, the physical mechanism of heterogeneous coupled heat transfer in the composite system, the atomic vibration power spectra of n-octadecane and graphene were calculated respectively in this section, the expression is as follows:

$$
I(\omega)=\operatorname{Re}\left[\frac{1}{2 \pi} \int_{0}^{\infty}\langle\vec{v}(t) \cdot \vec{v}(0)\rangle e^{-i w t} d t\right]
$$

Where $I(\omega)$ is the vibration power spectrum when the frequency is $\omega,\langle\vec{v}(t) \cdot \vec{v}(0)\rangle$ is velocity auto-correlation function. The vibration power spectrum of n-octadecane and graphene in the composite system at equilibrium state $280 \mathrm{~K}$ in Fig.1(d).

We can see that the atomic vibration power spectra of graphene and n-octadecane overlap a large part in the range of $20 \sim 43 \mathrm{THz}$, while the overlap of vibration modes will help heat transfer, which is conducive to improving the heat conduction between graphene and n-octadecane molecules in the system.Furthermore, The peak of graphene at $36 \mathrm{THz}$ is significantly higher than others, most of which comes from the vibration mode of atoms in the normal direction of the plane, indicating that the movement of graphene atoms outside the plane has better thermal coupling with n-octadecane.

\subsection{The radial distribution function}

The radial distribution function (RDF) is a mathematical language to describe the internal microstructure of the matter. The radial distribution function $g_{\alpha \beta}(r)$ represents the probability of the occurrence of a $\beta$ particle in a spherical shell volume $r \sim r+d r$ away from the reference $\alpha$ particle center by using the following equation:

$$
g_{\alpha \beta}(r)=\frac{d N}{\rho 4 \pi r^{2} d r}
$$

where $\rho$ is density of system, $N$ is the total number of particles in the system.
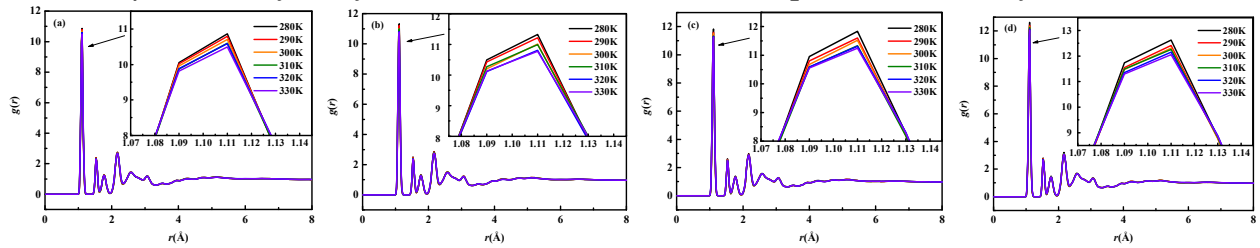

Fig. 3. Radial distribution function of n-octadecane molecules in each system at different temperatures.

Radial distribution functions of n-octadecane molecules in each system are shown in Fig.3.We can get that the radial distribution function only has peaks in the short range, showing the characteristics of short range order and long range disorder. The shape of the first peak indicates that the atomic number density within this radius is much higher than the average atomic number density of the system. By comparing the radial distribution function of each system, it was found that the peak value was higher as the mass fraction of graphene increased, which is due to the fact that the addition of graphene leads to a denser architecture, enhanced interaction with n-octadecane, and limited molecular diffusion.

\section{Conclusion}

Molecular dynamics simulation is applied to reveal crucial thermal properties, such as thermal conductivity, melting temperature and heat capacity, of three-dimensional 
nanoporous graphene/paraffin nanocomposites during phase transition. The results could draw the following conclusion.

1.The mean square displacement and self diffusion coefficients of atoms in each system increase with the increasing temperature, and the self diffusion coefficient changes with the temperature change rate near the phase transition point

2.The thermal conductivity of each system in solid state is higher than that in liquid state, and suddenly drops near the melting point

3.With the increasing temperature, the peak value of the radial distribution function gradually decreases and the width of the peak increases slightly, indicating that the thermal motion of atoms intensifies and the order degree of the system decreases.

\section{Acknowledgments}

This work is supported by the National Natural Science Foundation of China (No.51606017), the Youth Talent Support Program of Chongqing (CQYC2021059206), Chongqing Science Fund for Distinguished Young Scholars (cstc2021jcyj-jqX0015), and the Fundamental Research Funds for the Central Universities (No.106112016CDJXY145502).

\section{References}

1. Pielichowska K, Pielichowski K. Progress in materials science, 2014, 65: 67-123.

2. Sharma A, Tyagi V V, Chen C R, et al. Renewable and Sustainable energy reviews, 2009, 13(2): 318-345.

3. Lin Y, Jia Y, Alva G, et al. Renewable \& Sustainable Energy Reviews, 2018, 82.

4. Liu LK, Su D, Tang YJ, Fang GY. Renewable \& Sustainable Energy Reviews, 2016, 62:305-17.

5. Tay N H S, Liu M, Belusko M, et al. Renewable \& Sustainable Energy Reviews, 2016, 75.

6. Bugaje I M. International journal of energy research, 1997, 21(9): 759-766.

7. Velraj R, Seeniraj R V, Hafner B, et al. Solar energy, 1999, 65(3): 171-180.

8. Saha S K, Srinivasan K, Dutta P. Journal of Heat Transfer, 2008, 130(3): 034505.

9. Sedeh M M, Khodadadi J M. Carbon, 2013, 60: 117-128.

10. Sedeh M M, Khodadadi J M. Journal of Heat Transfer, 2014, 136(11): 112603.

11. Liu L, Su D, Tang Y, et al. Renewable and Sustainable Energy Reviews, 2016, 62: 305-317.

12. Novoselov K S, Geim A K, Morozov S V, et al. science, 2004, 306(5696): 666-669.

13. Ghosh S, Calizo I, Teweldebrhan D, et al. Applied Physics Letters, 2008, 92(15): 151911.

14. Geim A K, Novoselov K S. nature materials, 2007, 6:183-191

15. Novoselov K S, Fal V I, Colombo L, et al. nature, 2012, 490(7419): 192-200.

16. Balandin A A, Ghosh S, Bao W, et al. Nano Letters, 2008, 8(3): 902-907.

17. Chae H K,Siberio-Perez D Y, Kim J. Nature, 2004, 427(6974):523. 